\title{
Structural Design and PLC Control of the Machine Tool Manipulator
}

\author{
Lei Wang ${ }^{1, a}$ \\ ${ }^{1}$ School of Mechanical and Automotive Engineering, Anhui Polytechnic University, Wuhu 241000, \\ China \\ awangdalei2000@126.com
}

Keywords: machine tool manipulator, structural design, modeling, PLC control, simulation

\begin{abstract}
A kind of hydraulic-driven manipulator for machine tool is presented in this paper. Firstly, the manipulator's structure is designed and 3D model is built by using solidworks software. Secondly, the hydraulic system diagram for implementing the manipulator's sequences is established. Finally, control system of the machine tool manipulator is implemented based on PLC, and the experimental results show the correctness and feasibility of our design and control programs. Therefore, this can provide a certain referencing value for the practical production application.
\end{abstract}

\section{Introduction}

The industrial manipulator is a kind of automatic equipment, which can simulate the hand gestures of human been and achieve the automatic motion of grabbing, carrying and manipulation, according to the preset sequences, tracks and demands [1]. It is one of the most important equipments, which can realize the industrial production mechanization and automation $[1,2]$. Many applications demand manipulators with high degrees of repeatability, precision, and reliability. Therefore, some researches on the mechanical manipulator and its control have been done by many scholars [3-5]. In this paper, our designed the hydraulic-driven machine tool manipulator combined with $\mathrm{CNC}$ machine (machining centers, CNC milling machine, etc.) can form an automated production line to achieve processing (feeding, processing and automation of the cutting) without human interference.

\section{Structural Design}

The design technical parameters include: a) grasping weight: $5 \mathrm{~kg}$; b) degrees of freedom: 2 ; c) maximal work piece radius: $80 \mathrm{~mm}$. The movement parameters include: a) clamping stroke: $27 \mathrm{~mm}$; clamping speed: $0.054 \mathrm{~m} / \mathrm{s}$. b) stretching stroke: $60 \mathrm{~mm}$; stretching speed: $0.06 \mathrm{~m} / \mathrm{s}$. c) rotating range: $0^{\circ}-90^{\circ}$; rotating speed: $45^{\circ} / \mathrm{s}$. c) the finger gripping range: $60 \mathrm{~mm}-80 \mathrm{~mm}$. d) buffering mode: hydraulic buffer. e) driving mode: hydraulic driving; f) control mode: PLC control.

According to the above technical and movement parameters, the 3D model is shown in Fig.1. Our designed machine tool manipulator has two degrees of freedom, the one is for horizontal stretching out and the other is for rotating in the vertical plane for the work piece. The finger is driven to achieve loosening and clamping movements by the first hydraulic cylinder (gray hydraulic cylinder). The stretching movement is implemented by the second hydraulic cylinder (purple hydraulic cylinder). The rotating movement in the vertical plane is driven by the third hydraulic cylinder (yellow hydraulic cylinder).

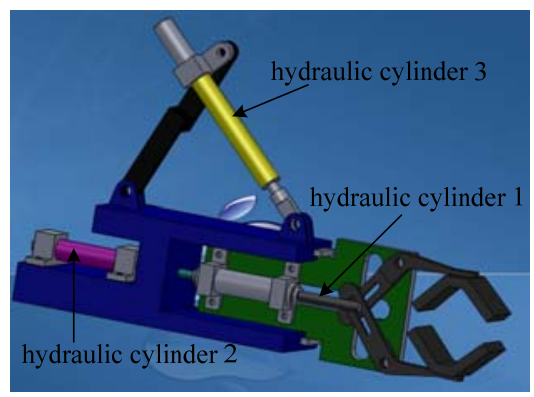

Fig.1 3D model of our designed manipulator 


\section{Hydraulic System Design and Implementation of Manipulator}

The operation sequences of the manipulator are: original position $\rightarrow$ telescopic boom falling $\rightarrow$ fingers clamping $\rightarrow$ telescopic boom retracting $\rightarrow$ telescopic boom upward swinging $\rightarrow$ telescopic boom stretching out $\rightarrow$ fingers loosening $\rightarrow$ fingers retracting $\rightarrow$ waiting for finishing machining $\rightarrow$ telescopic boom stretching out $\rightarrow$ fingers clamping $\rightarrow$ telescopic boom retracting $\rightarrow$ telescopic boom downward swinging $\rightarrow$ fingers loosening $\rightarrow$ telescopic boom rising to the original position and stopping, preparing for the next procedure.

According to the above operation sequences of the manipulator, the hydraulic principle diagram of the movements of the manipulator is established by using FluidSim software, as shown in Fig. 2.

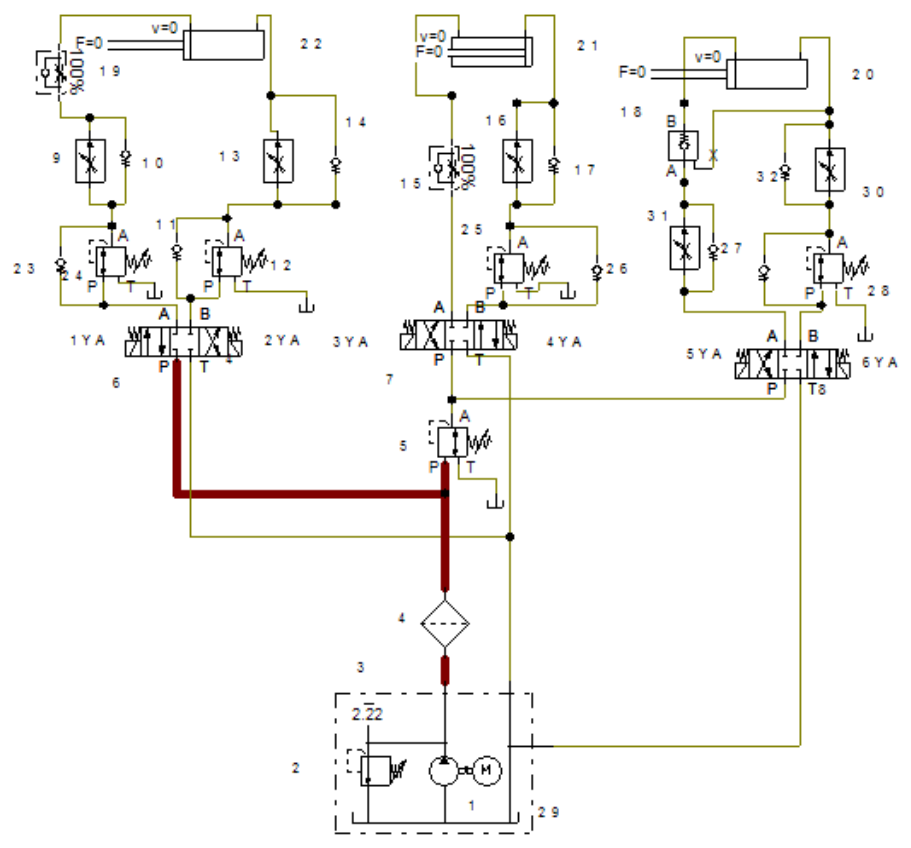

Fig.2 Hydraulic principle diagram

The manipulator's action sequences are shown in Table 1. The manipulator's stretching out and retracting movements are controlled by electromagnet 3YA, 4YA respectively, as shown in Fig.2. The manipulator's upward swinging and downward swinging movements are controlled by electromagnet 1YA, 2YA respectively. The manipulator's clamping and loosening movements are controlled by electromagnet 5YA, 6YA respectively. With the purpose of none mutual disturbances among multi-cylinder movements and realizing synchronous or nonsynchronous movements, three meso-position "O" mode electric-hydraulic reversing valves are adopted.

Table 1 the manipulator's action sequence table

\begin{tabular}{|c|c|c|c|c|c|c|c|}
\hline \multirow{2}{*}{$\begin{array}{c}\text { Serial } \\
\text { number }\end{array}$} & \multirow{2}{*}{ Action Name } & \multicolumn{7}{|c|}{ Electromagnet } \\
\cline { 2 - 8 } & original position & - & + & - & + & + & - \\
\hline 0 & stretching out & - & + & + & - & + & - \\
\hline 1 & clamping & - & + & + & - & - & + \\
\hline 2 & retracting & + & + & - & + & - & + \\
\hline 3 & upward swing & + & - & - & + & - & + \\
\hline 4 & stretching out & + & - & + & - & - & + \\
\hline 5 & loosening & + & - & + & - & + & - \\
\hline 6 & retracting & + & - & - & + & + & - \\
\hline 7 & stretching out & + & - & + & - & - & - \\
\hline 8 & clamping & + & - & + & - & + & + \\
\hline 9 & retracting & + & - & - & + & - & + \\
\hline 10 & downward swing & - & + & - & + & - & + \\
\hline 11 & loosening & - & + & - & + & + & - \\
\hline 12 & & & & & & + & + \\
\hline
\end{tabular}




\section{Control System Design and Simulation}

Programmable Logic Controller (PLC) is widely used in different areas [6, 7] including mechanical engineering, electrical engineering and so on because it has high reliability, intuitive programming, good adaptability, strong interface functions. Therefore, we also adopt "FX2N-16MR" mode PLC to control the machine tool manipulator. This type has 16 total numbers of I/O ports, namely, 8 inputs, 8 outputs. The I/O ports allocated in our control system are shown in Table 2. Fig.3 shows the experimental debugging process, the control programs are shown in Table 3, Fig. 4 shows the host computer's monitoring process. The repeated experimental results showed that the correctness of PLC-based control programs.

Table $2 \mathrm{I} / \mathrm{O}$ ports allocations

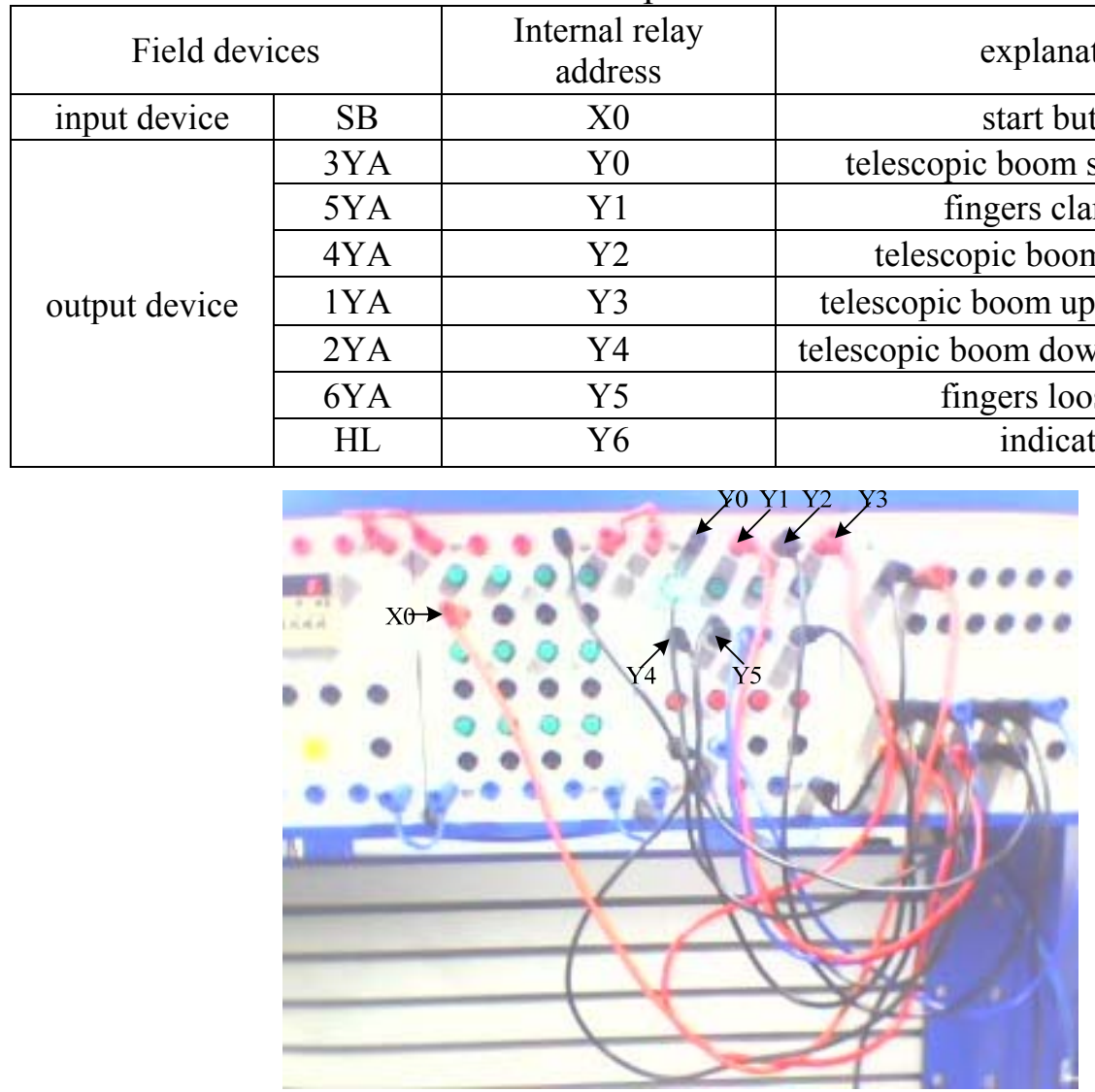

Fig.3 Experimental debugging process

Table 3 Instruction list

\begin{tabular}{|c|c|c|c|c|c|c|c|c|c|c|c|c|c|}
\hline 0 & LD & X001 & & 15 & OUT & T10 & K25 & 30 & ORB & & 45 & ANI & T5 \\
\hline 1 & OR & M0 & & 16 & OUT & T11 & K27 & 31 & OUT & Y001 & 46 & LD & T11 \\
\hline 2 & ANI & T12 & & 17 & OUT & T12 & K28 & 32 & LD & T1 & 47 & ANI & T12 \\
\hline 3 & OUT & M0 & & 18 & LD & T6 & & 33 & ANI & T2 & 48 & ORB & \\
\hline 4 & LD & M0 & & 19 & AND & T7 & & 34 & LD & T5 & 49 & OUT & Y004 \\
\hline 5 & OUT & T0 & K1 & 20 & ANI & T8 & & 35 & ANI & T6 & 50 & LD & T10 \\
\hline 6 & OUT & T1 & K2 & 21 & LD & T3 & & 36 & ORB & & 51 & ANI & T11 \\
\hline 7 & OUT & T2 & K3 & 22 & ANI & T4 & & 37 & LD & T9 & 52 & OUT & Y005 \\
\hline 8 & OUT & T3 & K5 & 23 & ORB & & & 38 & ANI & T10 & 53 & END & \\
\hline 9 & OUT & T4 & K6 & 24 & OR & T0 & & 39 & ORB & & 54 & & \\
\hline 10 & OUT & T5 & K7 & 25 & OUT & Y000 & & 40 & OUT & Y002 & 55 & & \\
\hline 11 & OUT & T6 & K8 & 26 & LD & T0 & & 41 & LD & T2 & 56 & & \\
\hline 12 & OUT & T7 & K22 & 27 & ANI & T1 & & 42 & ANI & T3 & 57 & & \\
\hline 13 & OUT & T8 & K23 & 28 & LD & T8 & & 43 & OUT & Y003 & 58 & & \\
\hline 14 & OUT & T9 & K24 & 29 & ANI & T9 & & 44 & LD & T4 & 59 & & \\
\hline
\end{tabular}




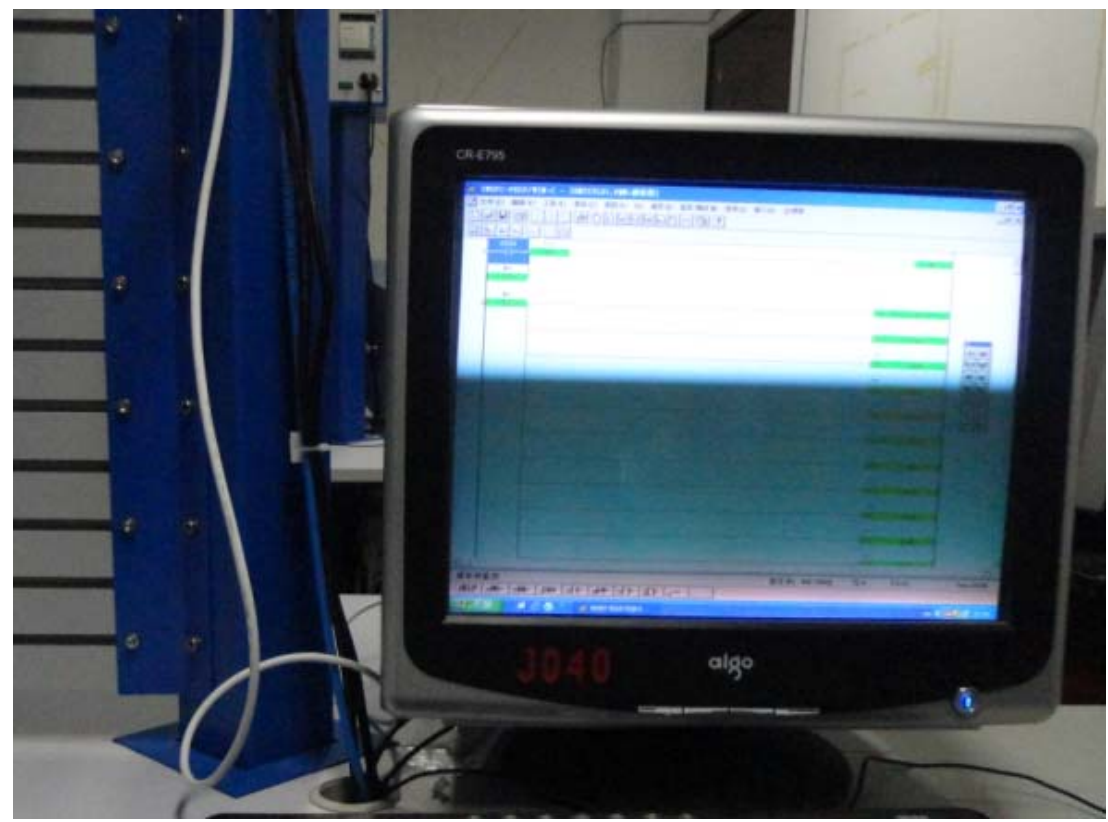

Fig.4 Host computer's monitoring process

\section{Conclusions and Future Work}

A kind of hydraulic-driven manipulator for machine tool is presented in this paper. Firstly, the manipulator's structure is designed and 3D models are built by using solidworks software. Secondly, the hydraulic system diagram for implementing the manipulator's sequences is established. Finally, control system of the machine tool manipulator is implemented based on PLC, and the experimental results show the correctness and feasibility of our design and control programs. Therefore, the results of the paper are very useful for the practical design and control of the device. The future work is how to use some good control algorithms to control the accuracy of movement of the machine tool manipulator.

\section{Acknowledgements}

This work is financially supported by the national natural science foundation of China (No. 51175262), Anhui provincial natural science foundation (No.1208085QE94), the doctoral scientific research starting foundation of Anhui Polytechnic University (No.2011YQQ006), universities natural science research project of Anhui province (No. KJ2012B008, No.KJ2010B021), and the youth foundation of Anhui Polytechnic University (No. 2012YQ29)

\section{References}

[1] W. Q. Hu, and L. H. Guo: Advances in Intelligent and Soft Computing Vol. 160(2012), p.615-619

[2] S.Wang, and H.Xu: Journal of Changchun Institute of Technology (Natural Science Edition) Vol.3 (2008), p.45-48

[3] J. S. Zhao, Z. J.Feng, L. P. Wang: Robotica Vol.24 (2006), p.635-641

[4] D. Kim and W. K. Chung: Journal of Mechanical Design, Vol. 125(2003), p.158-164

[5] Y. Li: Science and Technology Information Vol.18 (2008), p.30-31

[6] A. Ramirez-Serrano, S. C. Zhu, S. K. H. Chan, S. S. W. Chan, M. Ficocelli and B. Benhabib: Journal of Intelligent Manufacturing, Vol.13 (2002), p.261-281

[7] A.W.L. Yao, S.D. Strombeck and J.S.C. Chi: the International Journal of Advanced Manufacturing Technology, Vol. 25 (2005), p.723-729 